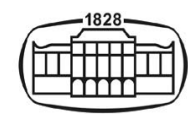

AKADÉMIAI KIADÓ

Acta Microbiologica et Immunologica Hungarica

67 (2020) 1, 23-32

DOI: 10.1556/030.66.2019.029

(c) 2019 Akadémiai Kiadó, Budapest

ORIGINAL ARTICLE

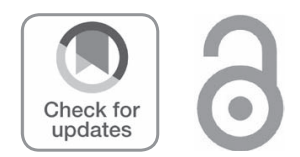

\section{Comparison of in vitro assays to study the effectiveness of antiparasitics against Acanthamoeba castellani trophozoites and cysts}

\author{
${ }^{1}$ Department of Ophthalmology, Saarland University Medical Center, Homburg/Saar, Germany \\ ${ }^{2}$ The First Affiliated Hospital of USTC, Division of Life Sciences and Medicine, University of Science and \\ Technology of China, Hefei, P. R. China \\ ${ }^{3}$ Institute for Medical Microbiology and Hygiene, Saarland University, Homburg/Saar, Germany \\ ${ }^{4}$ Department of Parasitology, National Public Health Center, Budapest, Hungary \\ ${ }^{5}$ Mycology and Parasitology Unit FG16, Robert Koch Institute, Berlin, Germany \\ ${ }^{6}$ Department of Ophthalmology, Semmelweis University, Budapest, Hungary
}

Received: May 19, 2019 • Accepted: July 12, 2019 • Published online: December 13, 2019
* Corresponding author: Dr. Lei Shi

Department of Ophthalmology, Saarland University Medical Center, Homburg/Saar, Germany; Department of Ophthalmology, The First Affiliated Hospital of University of Science and Technology of China, Hefei, China Phone: +49 68411622 387; Fax: +496841 1622400

E-mail: shileidr@outlook.com

\begin{abstract}
We aimed to compare LDH release assay, trypan blue and fluorescent stainings, and non-nutrient Escherichia coli plate assay in determining treatment efficacy of antiamoebic agents against Acanthamoeba castellanii trophozoites/cysts, in vitro. $1 \mathrm{BU}$ trophozoites/cysts were challenged with $0.02 \%$ polyhexamethylene biguanid (PHMB), $0.1 \%$ propamidine isethionate (PD), and $0.0065 \%$ miltefosine (MF). Efficacies of the drugs were determined by LDH release and trypan blue assays, by Hoechst 33343 , calcein-AM, and ethidium homodimer-1 fluorescent dyes, and by a non-nutrient agar E. coli plate assay. All three antiamoebic agents induced a significant LDH release from trophozoites, compared to controls $(p<0.0001)$. Fluorescent-dye staining in untreated 1BU trophozoites/cysts was negligible, but using antiamoebic agents, there was $59.3 \%-100 \%$ trypan blue, $100 \%$ Hoechst $33342,0 \%-75.3 \%$ calcein-AM, and $100 \%$ ethidium homodimer-1 positivity. On E. coli plates, in controls and MF-treated 1BU trophozoites/cysts, new trophozoites appeared within $24 \mathrm{~h}$, encystment occurred after 5 weeks. In PHMB- and PD-treated 1BU throphozoites/cysts, irregularly shaped, smaller trophozoites appeared after $72 \mathrm{~h}$, which failed to form new cysts within 5 weeks. None of the enzymatic- and dye-based viability assays tested here generated survival rates for trophozoites/cysts that were comparable with those yielded with the non-nutrient agar E. coli plate assay, suggesting that the culture-based assay is the best method to study the treatment efficacy of drugs against Acanthamoeba.
\end{abstract}

\section{KEYWORDS}

Acanthamoeba castellanii 1BU, LDH release assay, trypan blue assay, non-nutrient agar $E$. coli plate assay

\section{INTRODUCTION}

Acanthamoeba are free-living protozoans, which are ubiquitous in nature and can be isolated from soil and water including fresh water, sea water, swimming pool, and bottled water or even from air [1]. Acanthamoeba exists in two life forms: active trophozoites and dormant cysts. Cysts are smaller, round, double-walled, long-term stages, which are highly resistant to a stressful and extreme environment. Acanthamoeba cysts may remain dormant but viable for 
several years or even decades. Encystment occurs under stressful conditions, such as $\mathrm{pH}$ changes or alterations of oxygen or food supply [2-4]. Trophozoites are the infectious form, resulting in humans mainly in two types of infection: granulomatous amoebic encephalitis, a rare but often fatal infection of the central nervous system that predominantly occurs in immunocompromised individuals $[5,6]$, and Acanthamoeba keratitis (AK). AK is a relatively rare but serious sight-threatening corneal infection that may also occur in immunocompetent subjects. About $90 \%$ of AK patients are contact lens wearers [7], and with increasing use of contact lenses, the number of AK patients is likely to grow. Although AK patients need long-term treatment, due to the low number and heterogeneity of AK cases, no standardized treatment of AK has been established yet [8]. Up-to-date biguanides such as polyhexamethylene biguanide (PHMB) and chlorhexidine, diamidines such as propamidine isethionate (PD) and hexamidine, or the alkylphosphocholine analog miltefosine (MF) have been used as off-label drugs to treat $\mathrm{AK}$, and the antibiotic neomycin also showed some promising antiamoebic effect [9]. However, biguanides and diamidines do not only damage Acanthamoeba, but are also cytotoxic for human corneal epithelial cells, keratocytes, and endothelial cells [10]. The lack of a standardized therapy against $\mathrm{AK}$ is in part also due to the lack of a generally agreed drug-testing regime against Acanthamoeba. Although a number of staining methods and viability tests have been utilized already to test the efficacy of drugs against Acanthamoeba isolates in vitro [11-14], a direct comparison how these methods perform on healthy and drug-treated trophozoites and cysts is still missing.

To fill this gap, we compared a number of commonly used viability and life-dead staining assays such as the lactate dehydrogenase $(\mathrm{LDH})$ release assay, trypan blue and fluorescent stainings, and the non-nutrient agar Escherichia coli plate assay in combination with trypan blue staining in determining treatment efficacies of antiamoebic agents against Acanthamoeba castellanii isolate $1 \mathrm{BU}$ trophozoites and cysts in vitro.

\section{MATERIALS AND METHODS}

\section{Acanthamoeba isolate}

The A. castellanii strain 1BU was received from the Unit for Mycotic, Parasitic and Mycobacterial Infections (FG16) of the Robert Koch Institute, Berlin, Germany.

\section{Acanthamoeba cultures}

$1 \mathrm{BU}$ trophozoites were axenically grown in tissue culture flasks containing $5 \mathrm{ml}$ of peptone yeast glucose (PYG) broth medium [15] at $30{ }^{\circ} \mathrm{C}$, in an airtight container. Encystment was induced by adding Neff's constant-pH encystment medium [16] to trophozoites, instead of PYG broth medium, when trophozoites reached confluence.
Following incubation of the $1 \mathrm{BU}$ trophozoites in Neff's constant-pH encystment medium for 1 week at $30{ }^{\circ} \mathrm{C}$, cysts were harvested through three times repeated washing (PBS, Sigma-Aldrich, St. Louis, MO, USA) and centrifugation $(800 \times g)$ steps, then were resuspended in $5 \mathrm{ml}$ Page's amoeba saline (PAS) [17] with a concentration of $3.30 \times 10^{6}$ cysts $/ \mathrm{ml}$ and were stored at $4{ }^{\circ} \mathrm{C}$ until usage.

\section{Antiamoebic agents and their preparation}

We used antiamoebic agents, such as PHMB (Pharmacy of Saarland Medical University, Homburg/Saar, Germany), PD (Brolene, Patheon UK Ltd., Swindon, UK), and MF (Sigma-Aldrich). PHMB was received as a $20 \%$ solution, PD as $0.1 \%$ Brolene ${ }^{\circledR}$ eye drops, and MF in powdered form. These agents were dissolved or diluted in PYG medium for treatment of 1BU trophozoites, and in PBS for treatment of $1 \mathrm{BU}$ cysts (final concentrations were $0.1 \% \mathrm{PHMB}, 0.02 \% \mathrm{PD}$, and $0.0065 \% \mathrm{MF}$ ). The above concentrations were set analog to the clinically used concentrations in human AK treatment (eye drops; Table I). As controls, 1BU trophozoites were cultivated in PYG medium and $1 B U$ cysts in PBS.

\section{LDH release assay (cytotoxicity)}

LDH activity in the supernatants of untreated and drugchallenged Acanthamoeba was determined with the CytoTox $96^{\circledR}$ Non-Radioactive kit (Promega Corporation, Madison, USA). For the determination of the cytotoxic effects of antiamoebic drugs on $A$. castellanii trophozoites, $1 \times 10^{4}$ 1BU trophozoites were incubated in 96-well plates with $100 \mu \mathrm{l} \mathrm{PYG}$ medium/well at $30{ }^{\circ} \mathrm{C}$ for $24 \mathrm{~h}$. During this time, 1BU trophozoites attached to the plate bottom and reached confluence. After $24 \mathrm{~h}$, PYG medium was replaced by $100 \mu \mathrm{l}$ of the antiameobic agent-containing medium (preparation described above) and the throphozoites were incubated for $2 \mathrm{~h}$ at $30^{\circ} \mathrm{C}$.

For the determination of the cytotoxic effects of antiamoebic drugs on $A$. castellanii cysts, $2 \times 10^{4} 1 \mathrm{BU}$ cysts were incubated in 96-well plates with $100 \mu \mathrm{l}$ antiamoebic agent containing medium at $30{ }^{\circ} \mathrm{C}$ for $2 \mathrm{~h}$. Negative controls received at the same time point $100 \mu \mathrm{l}$ of fresh PYG medium, and positive controls were incubated with $100 \mu$ l of fresh PYG supplemented with $10 \mu$ lysis solution (provided by the kit), and controls were also incubated at $30{ }^{\circ} \mathrm{C}$ for $2 \mathrm{~h}$. Thereafter, 96-well plates were centrifuged at $200 \times g$ (trophozoites) and $400 \times g$ (cysts) for $5 \mathrm{~min}$, respectively, and $50 \mu \mathrm{l}$ of the supernatants were removed from each well

Table I. Antiamoebic agent concentrations in our experiments

\begin{tabular}{|l|c|}
\hline Agent & Concentration \\
\hline Polyhexamethylen biguanid (PHMB) & $1079 \mu \mathrm{M}(0.02 \%)$ \\
\hline Propamidin isethionate (PD) & $1771 \mu \mathrm{M}(0.1 \%)$ \\
\hline Miltefosine (MF) & $160 \mu \mathrm{M}(0.0065 \%)$ \\
\hline
\end{tabular}


and transferred into the wells of a fresh 96-well plate. An amount of $50 \mu \mathrm{l}$ of substrate reagent was added to each well and the solution was carefully mixed and was incubated for $30 \mathrm{~min}$ at room temperature. The reaction was stopped by adding $50 \mu \mathrm{l}$ stop solution to each well. The LDH-catalyzed formazan formation was determined spectroscopically with a multimode microplate reader (PerkinElmer Ensight ${ }^{\mathrm{TM}}$, USA) at $490 \mathrm{~nm}$ that was blanked with medium and antiamoebic agent containing medium, respectively. Cytotoxicity (\%) was determined as follows:

$$
\begin{aligned}
& \text { Cytotoxicity }(\%)=100 \times\left(\text { Experimental LDH release } \mathrm{OD}_{490}\right. \\
& \text { - Blank control } \left.\mathrm{OD}_{490}\right) /\left(\text { Maximum LDH release } \mathrm{OD}_{490}\right. \\
& \text { - Blank control } \left.\mathrm{OD}_{490}\right)
\end{aligned}
$$

\section{Trypan blue assay}

About $2 \times 10^{4} 1 \mathrm{BU}$ trophozoites or cysts were suspended in $100 \mu \mathrm{l}$ of PYG or antiamoebic agent-containing medium (preparation described above for each agent) in $1.5 \mathrm{ml}$ reaction tubes (Sarstedt, Nümbrecht, Germany) and incubated in a Thermomixer (Eppendorf AG, Hamburg, Germany) with a shaking rate of $650 \mathrm{rpm}$ at $30{ }^{\circ} \mathrm{C}$ for $2 \mathrm{~h}$. Thereafter, all reaction tubes were centrifuged at $200 \times g$ for $5 \mathrm{~min}$ and $80 \mu \mathrm{l}$ supernatant was removed from each tube. As a next step, $20 \mu \mathrm{l}$ of a $0.4 \%$ trypan blue solution (Sigma-Aldrich) was added to each tube and throphozoites or cysts were incubated at room temperature for $5 \mathrm{~min}$. $1 \mathrm{BU}$ trophozoites and cysts without trypan blue staining served as negative controls. Thereafter, $10 \mu \mathrm{l}$ of the trypan blue treated cells were pipetted to a hemocytometer (C-Chip, NanoEnTek, Waltham, USA) and bright field images were taken with a Leica DMI4000 B microscope (Leica Microsystems, Wetzlar, Germany) at 10-fold magnification, using Leica Application Software v3.7. Using Image J (National Institute of Health, USA), we analyzed gray value for each blue-stained cell. Unstained cells in control group and other treated groups were not included. Then, we calculated the mean gray value for all blue-stained cells in each group separately and defined the gray value for each blue-stained cell, compared to the mean value as percentage. With this method, we summarized the cell amount in gray value ranges $85 \%-90 \%$, 90\%-95\%, and >95\%.

\section{Fluorescent staining}

1BU trophozoites or cysts $\left(2 \times 10^{4}\right.$ cells/well $)$ were resuspended in $100 \mu \mathrm{l}$ PYG (trophozoites) or PBS (cysts) in absence or presence of the antiamoebic agents, and were pipetted into a 96-well plate, which was subsequently incubated for $2 \mathrm{~h}$ at $30{ }^{\circ} \mathrm{C}$. As a positive control, primary human corneal keratocytes $\left(10^{4}\right.$ cells/well $)$ were resuspended in DMEM/F-12 medium (Thermofisher, Paisley, UK), pipetted into 96-well plates, and incubated for $2 \mathrm{~h}$ at $37^{\circ} \mathrm{C}$.

$1 \mathrm{BU}$ containing 96-well plates were subsequently centrifuged at $200 \times g$ (trophozoites) or $400 \times g$ (cysts) for $5 \mathrm{~min}$, to remove the antiamoebic agent-containing media.

As a next step, cells were resuspended in $100 \mu \mathrm{l}$ of fluorescent stain solutions [Hoechst $33342(10 \mu \mathrm{g} / \mathrm{ml})$ or a mixture of calcein-AM $(4 \mu \mathrm{M})$ and ethidium homodimer-1 $(1 \mu \mathrm{M})]$ and incubated in the well plates for $30 \mathrm{~min}$ at room temperature in the dark. Thereafter, wells were washed three times with PBS to remove unbound fluorescent dyes, and cells were resuspended in PBS. Cell images were taken with an inverted fluorescence microscope (Leica Microsystems) using the following settings. Hoechst 33342 fluorescence was excited at $340 \mathrm{~nm}$ and the light emission determined at $510 \mathrm{~nm}$, calcein-AM was excited at $490 \mathrm{~nm}$ and light emission determined at $515 \mathrm{~nm}$, and ethidium homodimer-1 was excited at $493 \mathrm{~nm}$ and light emission determined at $617 \mathrm{~nm}$.

Hoechst 33343 stains nuclei of dead and viable cells to blue color, calcein-AM stains cytoplasm of viable cells to green color, and ethidium homodimer-1 stains nuclei of dead cells to red color.

\section{Non-nutrient agar E. coli plate assay}

The non-nutrient agar E. coli plate assay was carried out essentially as described by Narasimhan [18] and Kowalski [19]. First, E. coli strain IM08B [20] was grown overnight on sheep blood agar plates (Becton Dickinson, Heidelberg, Germany) at $35{ }^{\circ} \mathrm{C}$. Fresh grown IM08B colonies were picked with a cotton swab and suspended in PAS, equivalent to a McFarland of 4 . An amount of $50 \mu \mathrm{l}$ of this suspension was pipetted onto the surfaces of non-nutrient agar plates, and spread out using an L-shaped spreader. In a second step, $2 \times 10^{4}$ trophozoites or cysts were mixed with $100 \mu \mathrm{l}$ medium (PYG for trophozoites and PBS for cysts) in absence and presence of the antiamoebic agents, and incubated at $30{ }^{\circ} \mathrm{C}$ for $2 \mathrm{~h}$ in a Thermomixer (Eppendorf AG) with a shaking rate of $650 \mathrm{rpm}$. An amount of $10 \mu \mathrm{l}$ of the cell suspensions was transferred into fresh tubes, mixed with $10 \mu \mathrm{l}$ of a trypan blue solution $(0.4 \%)$ and incubated for $5 \mathrm{~min}$ at room temperature. An amount of $980 \mu \mathrm{l}$ of PBS was subsequently added to each tube (the drugs were diluted 100 folds) and the cells were carefully suspended by pipetting gently up and down. An amount of $50 \mu \mathrm{l}$ of the stained cell suspensions (100 cells) was next pipetted into the middle of the non-nutrient agar E. coli plates, and the suspension desiccated for around $10 \mathrm{~min}$. Next, circles were drawn on the bottom of the plates to confine the borders of the Acanthamoeba cell solution, and bright field images of these regions were taken. A. castellanii inoculated plates were incubated upside up for $24 \mathrm{~h}$ at $30{ }^{\circ} \mathrm{C}$. Thereafter, plates were sealed by parafilm (Pechiney, Menasha, USA) and were incubated upside down for up to 5 weeks at $30{ }^{\circ} \mathrm{C}$.

Bright field images were taken with a Leica DMI4000 B microscope every $24 \mathrm{~h}$ until $72 \mathrm{~h}$, then every week until the end of the 5 th week, using a 10 -fold magnification. These experiments were repeated three times on different days. 


\section{Statistical analysis}

The results of the LDH release (cytotoxicity) and trypan blue assays were statistically analyzed using one-way analysis of variance followed by Tukey's multiple comparisons test (to compare the effect of each antiamoebic agent).

\section{RESULTS}

\section{LDH release assay}

To confirm the antiamoebic potentials of the drugs PHMB (0.02\%), PD (0.1\%), and MF (0.0065\%) on Acanthamoeba trophozoites $[18,19,21]$, a series of $\mathrm{LDH}$ release assays were carried out (Figure 1). All antiamoebic agents induced a significant release of $\mathrm{LDH}$ into the supernatants of the drug-challenged trophozoite cultures when compared to the unchallenged control group $(p<0.0001)$, in line with previous studies reporting that all three drugs exhibited a significant cytotoxicity on Acanthamoeba trophozoites [22]. Based on the $\mathrm{LDH}$ release values, MF was identified as the most effective drug of this test panel causing a significantly higher LDH release than PD $(p=0.0011)$ and PHMB $(p<0.0001)$. $\mathrm{PD}$ was significantly more effective in releasing $\mathrm{LDH}$ than PHMB $(p=0.0004)$. As the lysis buffer supplied by the CytoTox $96{ }^{\circledR}$ Non-Radioactive kit could not lyse 1BU cysts, we could not set a maximum $\mathrm{LDH}$ release control for these and we could only perform LDH assay for 1BU trophozoites.

\section{Trypan blue assay}

The effects of PHMB, PD, and MF on the viability of $1 \mathrm{BU}$ cells were next determined with a trypan blue exclusion assay [23], which monitors the integrity of cell membranes (Figure 1 and the corresponding phase-contrast images of Figures 2 and 3). In untreated 1BU trophozoites and cysts (controls), we observed only a small proportion of cells that displayed a relevant blue staining. Following trypan blue

Figure 1. Cytotoxic effect of $0.02 \% \mathrm{PHMB}, 0.1 \% \mathrm{PD}$, and $0.0065 \% \mathrm{MF}$, on $1 \mathrm{BU}$ trophozoites and cysts, using trypan blue assay $(n=3)$ and on 1BU trophozoites, using $L D H$ assay $(n=5)$. Trypan blue assay: $0.02 \%$ PHMB, $0.1 \%$ PD, and $0.0065 \%$ MF had a significant cytotoxic effect on $1 \mathrm{BU}$ trophozoites and cysts $(p<0.0001) .0 .1 \% \mathrm{PD}$ and $0.0065 \% \mathrm{MF}$ had a significantly higher cytotoxic effect on $1 \mathrm{BU}$ trophozoites than $0.02 \%$ PHMB $(p<0.0001)$. There was no significant difference between $0.1 \%$ PD and $0.0065 \%$ MF cytotoxicity for 1 BU trophozoites $(p=0.0608)$. The cytoxic effect on $1 \mathrm{BU}$ cysts was significantly less using $0.0065 \% \mathrm{MF}$ than $0.02 \%$ PHMB or $0.1 \%$ PD $(p<0.0001)$. However, there was no significant difference between cytotoxic effect of $0.02 \%$ PHMB and $0.1 \%$ PD, observing $1 B U$ cysts $(p=0.9883)$. LDH assay: $0.0065 \%$ MF was significantly more cytotoxic than $0.1 \%$ PD $(p=0.0011)$ and $0.02 \%$ PHMB $(p<0.0001)$, and $0.1 \%$ PD was significantly more cytotoxic than $0.02 \%$ PHMB $(p=0.0004)$, observing $1 \mathrm{BU}$ trophozoites

Trypan blue assay
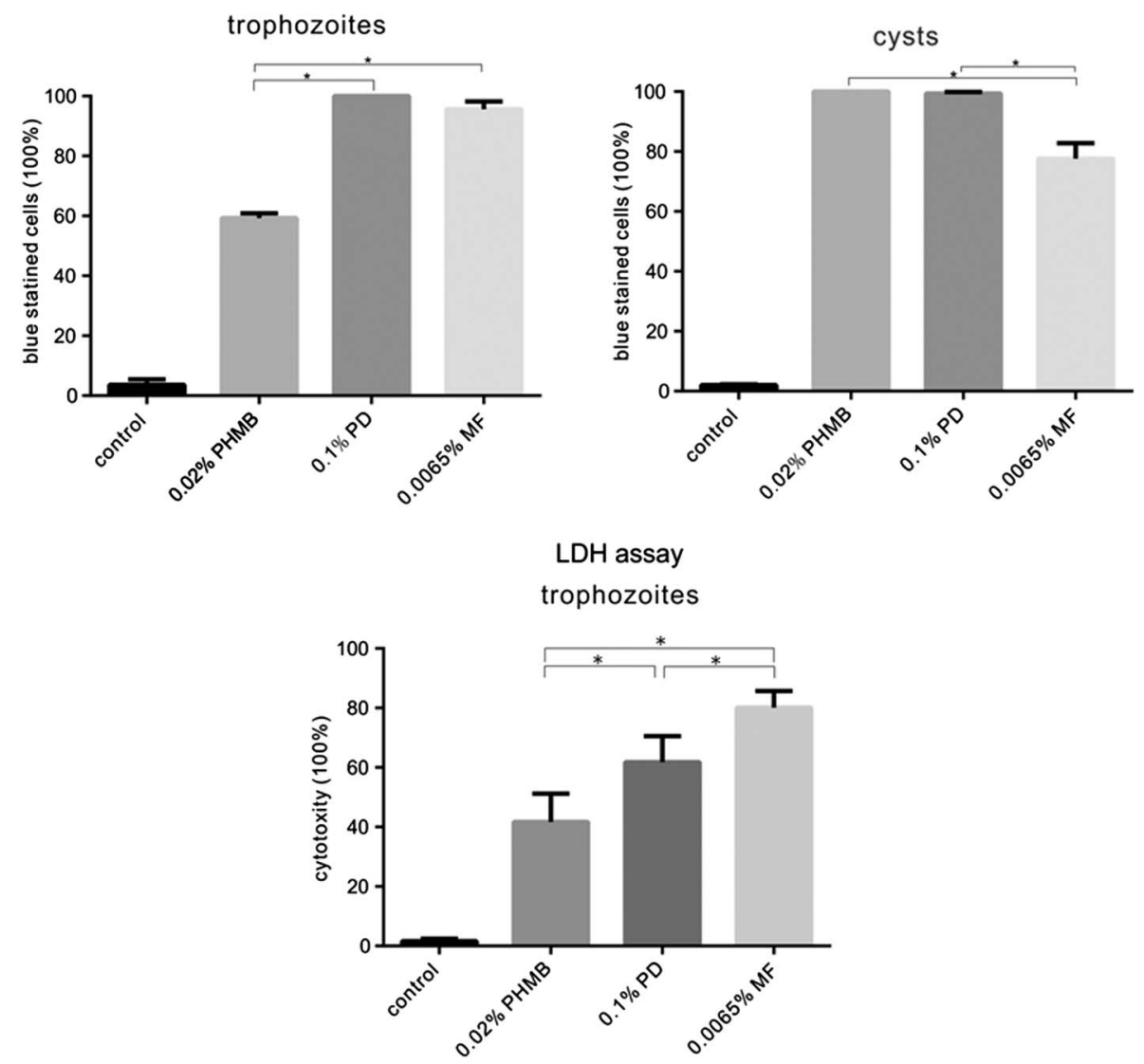
staining, about $3.7 \% \pm 1.8 \%$ of the drug-free trophozoites and $2.1 \% \pm 0.2 \%$ of the cysts displayed a clear blue signal, suggesting that these cells featured compromised cell membranes/cyst walls. However, challenging 1BU cells with the antiamoebic drugs clearly increased the number of trypan blue positive cells for all three drugs tested (Figure 1 and
Table II). Challenging 1BU cells with PHMB clearly increased the ratios of blue stained cells to $59.3 \% \pm 1.5 \%$ (trophozoites) and $100 \%$ (cysts), respectively, suggesting that the membranes/cyst walls of the majority of PHMB-treated cells became permeable for trypan blue by this drug. This effect was even stronger when $1 \mathrm{BU}$ cells were challenged with

Figure 2. Trypan blue, Hoechst 33342, calcein-AM and ethidium homodimer-1 staining of 1BU trophozoites, with and without antiamoebic agent tretament. Hoechst 33342, calcein-AM, and ethidium homodimer-1 did not stain untreated trophozoites and cysts. Trypan blue stained 3.6\%, Hoechst 33342 100\%, ethidium homodimer-1 100\%, calcein-AM $75.33 \%$ of $0.02 \%$ PHMB-treated trophozoites. Following $0.1 \%$ PD treatment, Hoechst 33342 and ethidium homodimer-1 were $100 \%$, trypan blue 59.33\%, calcein-AM 9.67\% positive in trophozoites. $0.0065 \%$ MF exposure resulted in $100 \%$ Hoechst 33342 and $100 \%$ ethidium homodimer-1, 95.67\% trypan blue and in $0 \%$ calcein-AM positivity of trophozoites

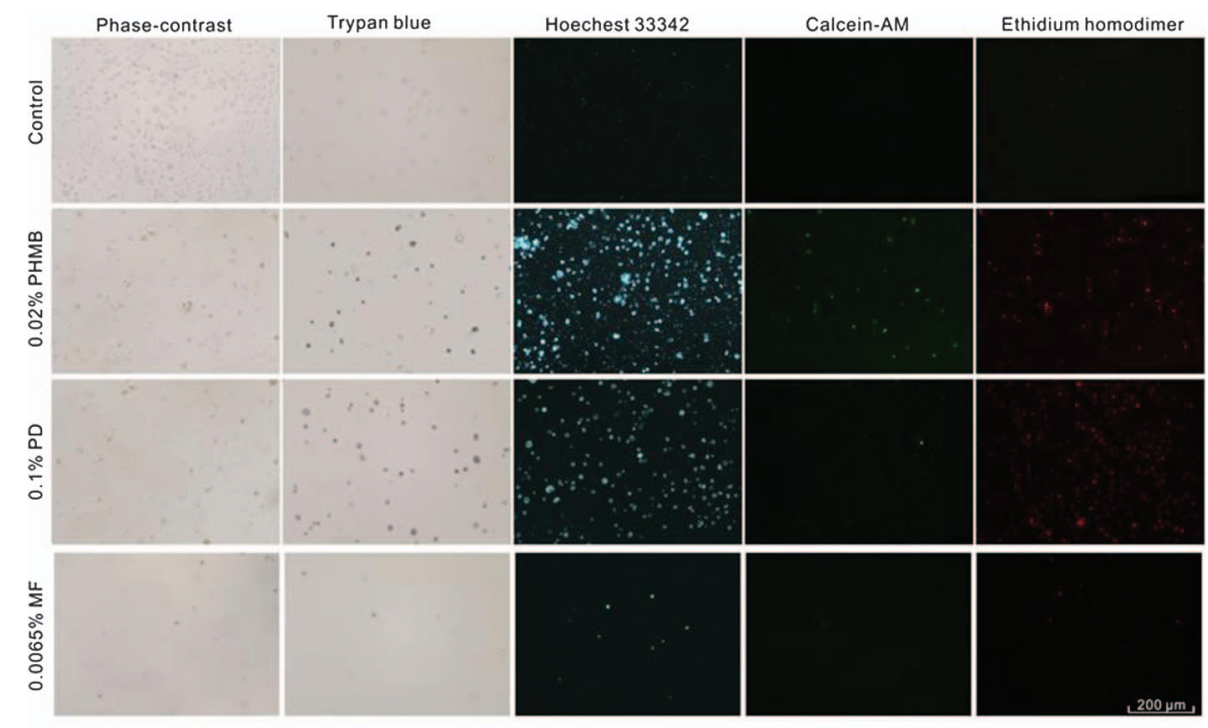

Trophozoites

Figure 3. Trypan blue, Hoechst 33342, calcein-AM and ethidium homodimer-1 staining of 1BU cysts, with and without antiamoebic agent tretament. Hoechst 33342, calcein-AM, and ethidium homodimer-1 did not stain untreated cysts. Trypan blue stained $100 \%$, Hoechst 33342 $100 \%$, ethidium homodimer-1 100\%, calcein-AM 60.67\% of $0.02 \%$ PHMB-treated cysts. Following $0.1 \%$ PD treatment, Hoechst 33342 and ethidium homodimer-1 were $100 \%$, trypan blue $99.33 \%$, and calcein-AM $36.67 \%$ positive in cysts. $0.0065 \%$ MF exposure resulted in $77.67 \%$ trypan blue, 100\% Hoechst 33342 and $100 \%$ ethidium homodimer-1, and in $0 \%$ calcein-AM positivity of cysts

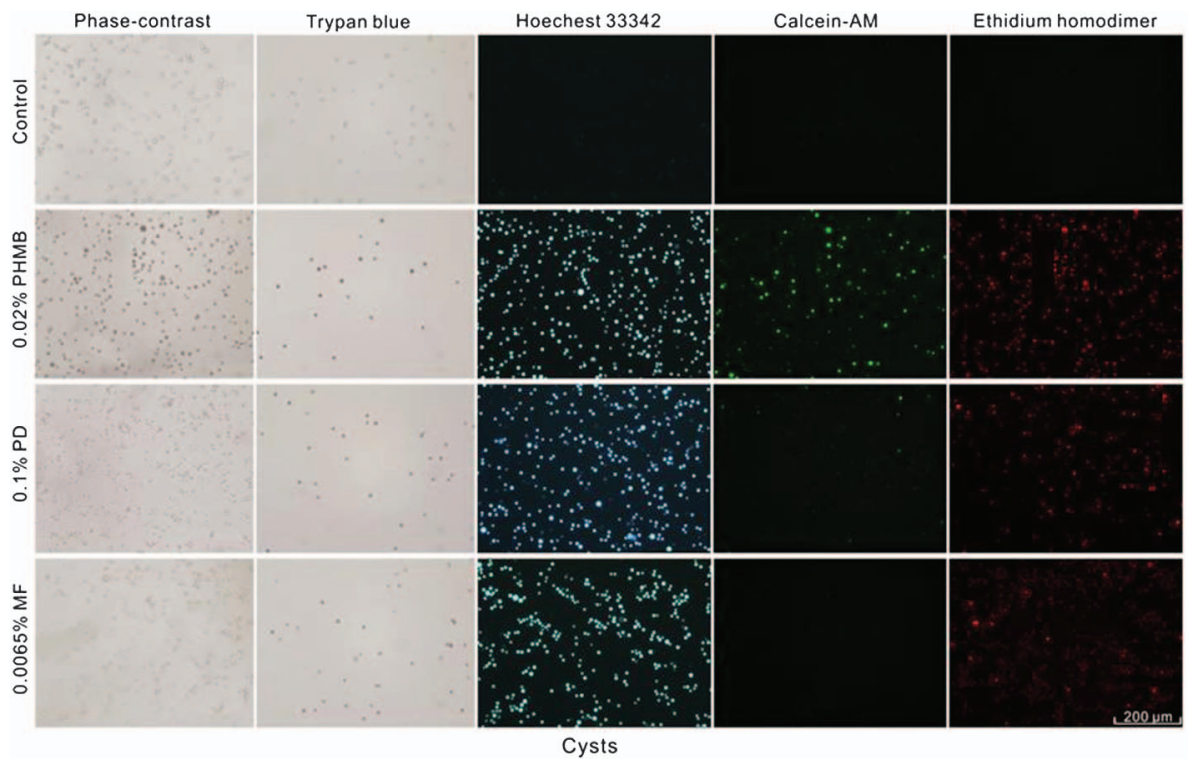


PD and MF, respectively. The latter drug increased the ratios of blue-stained cells to $100 \%$ (trophozoites) and $77.7 \% \pm 5.1 \%$ (cysts), while almost all PD-treated $1 \mathrm{BU}$ trophozoites/cysts became trypan blue-positive (Table II). These findings are basically in line with the LDH release assay results (Figure 1), suggesting that all tested drugs exerted highly significant cytotoxic effects on 1BU cells, with $\mathrm{PD}$ and MF displaying very strong effects on trophozoites, while $\mathrm{PHMB}$ was significantly less effective in destabilizing trophozoites membranes (Figure 1). PHMB and PD exerted the strongest cytotoxic effect on cysts, while MF was significantly less effective against cysts (Figure 1). Interestingly, when drug-challenged cysts were screened for blue-staining intensities, some differences between drugs were noticed. Challenging cysts with PD yielded a highly homogeneous trypan blue-staining pattern with all cysts displaying mean gray values $>95 \%$. However, when the cysts were treated with MF and PHMB, respectively, a more heterogeneous staining pattern was observed. MF and PHMB produced small proportions of blue-stained cysts that showed lower gray values (3.45\% with $85 \%-90 \%$, 6.90\% with $90 \%-95 \%$, and $89.65 \%$ with $>95 \%$ mean gray value for PHMB, and $9.1 \%$ with $90 \%-$ $95 \%$, and $90.9 \%$ with $>95 \%$ mean gray value for $\mathrm{MF}$ ).

\section{Fluorescent staining}

The impact of the antiamoebic drugs on cell viability of $A$. castellanii trophozoites and cysts was next tested with a small set of fluorescent dyes. Hoechst 33342 is a cell permeable blue fluorescent dye that is specific for DNA and can be used to stain the nuclei of viable and fixed cells $[24,25]$. The cell permeable vital stain calcein-AM is a non-fluorescent derivative of the green fluorescent dye calcein, which can be converted to calcein due to the activity of cytosolic esterases [26]. The vital stain is often used in combination with ethidium homodimer-1, a cell impermeable DNA-specific redfluorescent dye that stains nuclei of dead cells [27]. Drugtreated trophozoites and cysts and healthy controls were mixed either with Hoechst 33342 or a combination of calcein-AM and ethidium homodimer-1, and fluorescence images were taken as outlined in "Materials and Methods" section. Representative fluorescence images of Hoechst 33342 and calcein-AM/ethidium homodimer-1 treated cells are displayed in Figures 2 and 3 , respectively, and percentages of Hoechst 33342, calcein-AM, and ethidium homodimer-1-positive trophozoites/cysts are presented in Table II. Notably, neither Hoechst 33342 nor the calcein-AM/ethidium homodimer-1 mixture stained drug-free trophozoites and cysts, indicating that both cell permeable dyes are not readily taken up and/or converted to a fluorescent dye by both $A$. castellanii growth stages. However, this changed when $1 B U$ cells were challenged with the antiamoebic drugs. Hoechst 33342 and ethidium homodimer-1 stained 100\%/ $100 \%$ of the PHMB-treated $1 \mathrm{BU}$ cells blue and red, respectively, and calcein-AM stained $75.3 \% \pm 4.0 \% / 60.7 \% \pm 3.5 \%$ of the PHMB-challenged trophozoites/cysts green. Following PD treatment, Hoechst 33342 and ethidium homodimer-1 were $100 \% / 100 \%$ positive in trophozoites/cysts, and calceinAM stained $9.7 \% \pm 2.5 \% / 36.7 \% \pm 4.5 \%$ of the $\mathrm{PD}$-treated

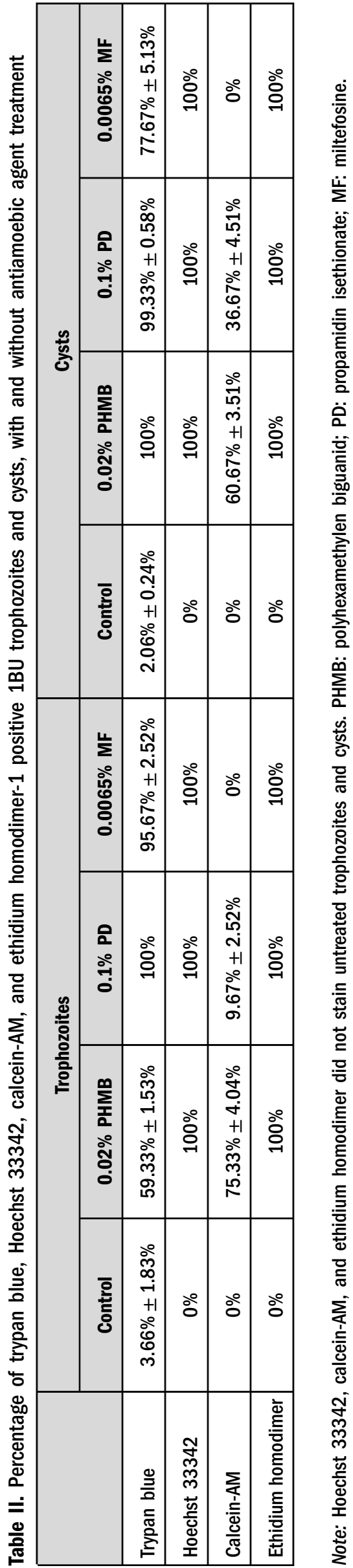


trophozoites/cysts green. Similarly, MF exposure yielded in 100\%/100\% Hoechst 33342 and ethidium homodimer-1 stained trophozoites/cysts. However, none of the MF-treated $1 \mathrm{BU}$ trophozoites/cysts emitted relevant amounts of green fluorescence upon exposure to light of the wavelength $490 \mathrm{~nm}$, indicating that MF-treated A. castellanii trophozoites and cysts cannot convert the non-fluorescent calcein-derivative calcein-AM to the green fluorescent calcein. Surprisingly, $75.3 \% \pm 4.0 \% / 60.7 \% \pm 3.5 \%$ of the PHMB-challenged trophozoites/cysts were positive for both ethidium homodimer-1 and calcein, suggesting that the membranes or cyst walls of the drug-challenged 1BU cells were sufficiently compromised to allow the diffusion of ethidium homodimer-1 into the cells but were viable enough to convert calcein-AM to calcein. Similar results were observed with PD-treated $1 \mathrm{BU}$ cells, which were in $9.7 \% \pm 2.5 \%$ (trophozoites) and $36.7 \% \pm 4.5 \%$ (cysts) positive for both calcein and ethidium homodimer-1. The fact that PHMB- and PD-challenged cysts were stained green in presence of calcein-AM indicates furthermore that cysts treated with these drugs can convert calcein-AM to calcein, suggesting that $A$. castellanii cysts contain sufficient amounts of active non-specific esterases to cleave the acetoxymethyl esters from the non-fluorescent calcein derivative to generate a fluorescent probe.

\section{Non-nutrient agar $E$. coli plate assay}

The aforementioned enzyme- and cell permeability-based viability assays suggested that the antiamoebic drugs $\mathrm{PHMB}$, $\mathrm{PD}$, and MF can kill significant amounts of, if not all, $A$. castellanii trophozoites and cysts under in vitro conditions. To confirm that these high killing rates suggested by the aforementioned assays are real, a non-nutrient agar E. coli plate assay was next carried out, in which trophozoites fed on an E. coli lawn. This assay also allows to monitor whether drug-challenged cysts can still germinate in a growthfavoring environment, and if cysts can be formed once the E. coli lawn was consumed by viable trophozoites. In this assay, untreated and drug-challenged trophozoites/cysts were stained by trypan blue and subsequently placed in the center of a non-nutrient agar plate inoculated with a layer of viable E. coli cells, and the germination of cysts and the growth of trophozoites were monitored over time by light microscopy. Representative phase-contrast images of $1 \mathrm{BU}$ trophozoites and cysts that were cultivated for 0,3 , and 35 days on nonnutrient agar E. coli plates are shown in Figures 4 and 5. For both 1BU trophozoites and cysts, the trypan blue staining disappeared within $24 \mathrm{~h}$ and therefore a follow-up of the fate of trypan blue-positive Acanthamoebae beyond this time frame was not possible.

Figure 4. Phase-contrast images of $1 \mathrm{BU}$ trophozoites on non-nutrient agar $E$. coli plates, 0 and $72 \mathrm{~h}$ and 5 weeks following $0.02 \% \mathrm{PHMB}$, $0.1 \% \mathrm{PD}$, and $\mathbf{0 . 0 0 6 5 \%} \mathrm{MF}$ treatment and trypan blue staining. In controls and in $\mathbf{0 . 0 0 6 5 \%}$ MF-treated $1 \mathrm{BU}$ trophozoites groups, new trophozoites appeared outside than marked circle after $72 \mathrm{~h}$, and encystment could be observed following 5 weeks. In $0.02 \%$ PHMB- and 0.1\% PD-treated 1BU trophozoites groups, strange shaped trophozoites appeared after $72 \mathrm{~h}$ (arrows), which did not form cysts following 5 weeks. The strange trophozoites were smaller and thinner compared to the normal trophozoites

5 weeks

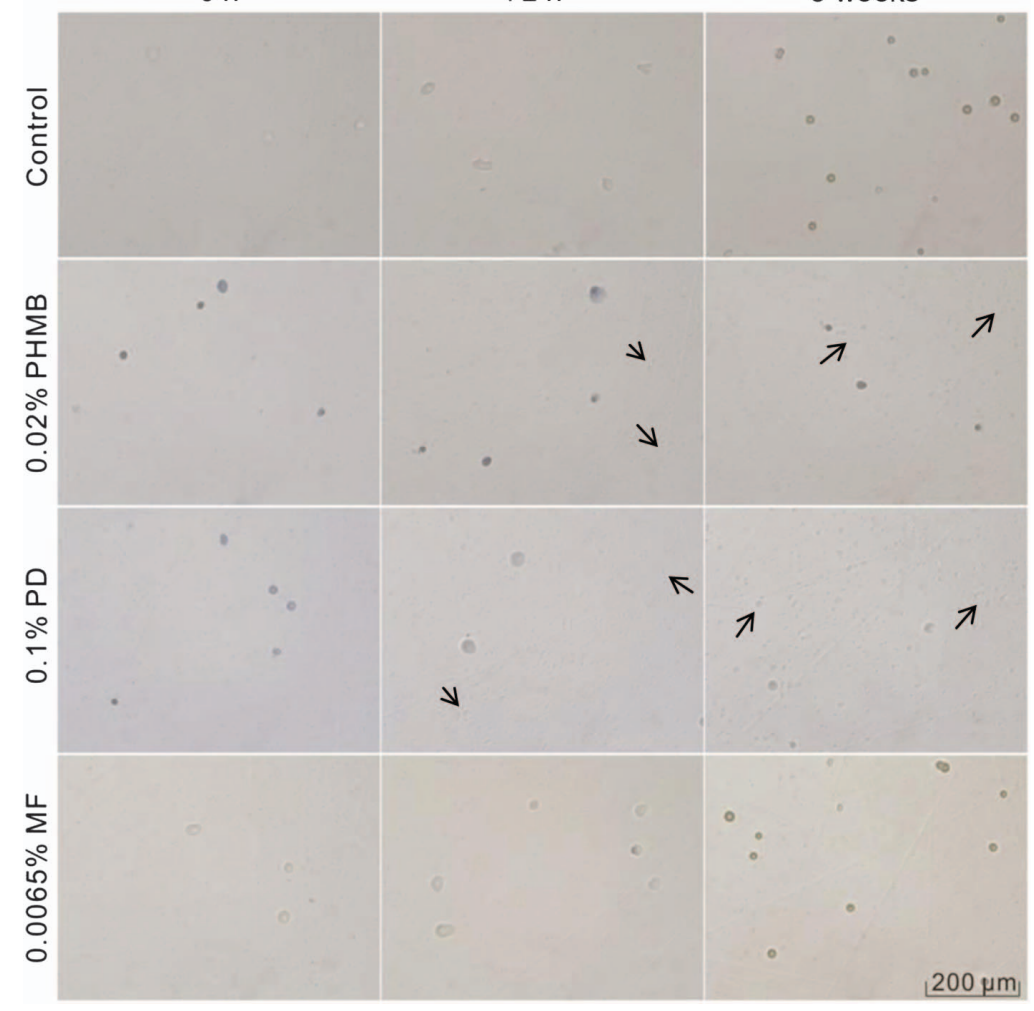


Figure 5. Phase-contrast images of $1 \mathrm{BU}$ cysts on non-nutrient agar $E$. coli plates, 0 and $72 \mathrm{~h}$ and 5 weeks following $0.02 \%$ PHMB, $0.1 \%$ $\mathrm{PD}$, and $\mathbf{0 . 0 0 6 5 \%} \mathrm{MF}$ treatment and trypan blue staining. In controls and in $\mathbf{0 . 0 0 6 5 \%}$ MF-treated $1 \mathrm{BU}$ cyst groups, trophozoites appeared after $72 \mathrm{~h}$, and encystment could be observed following 5 weeks. In 0.02\% PHMB- and 0.1\% PD-treated 1BU cyst groups, strange shaped trophozoites appeared after $72 \mathrm{~h}$ (arrows, see also Figure 6), which did not form cysts following 5 weeks. The strange trophozoites were smaller and thinner compared to the normal trophozoites

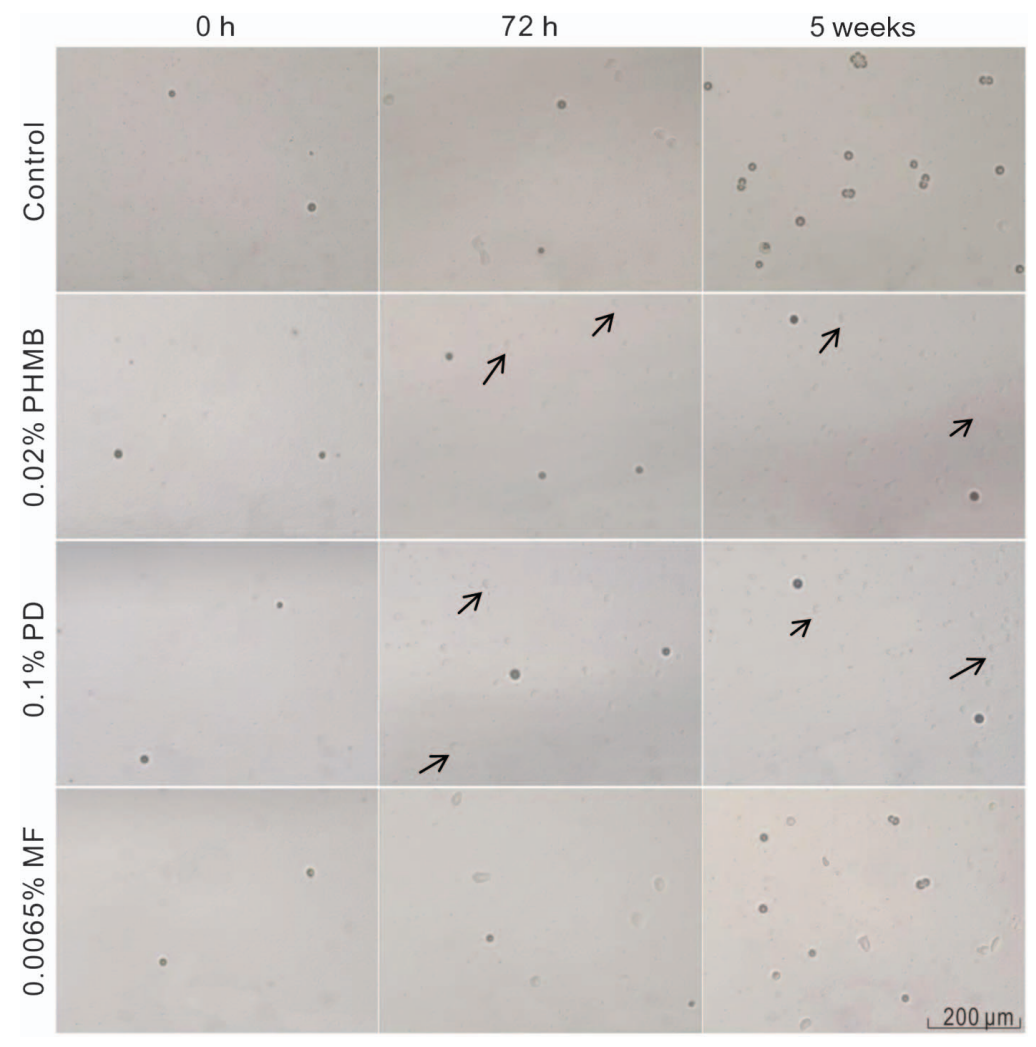

In drug-free controls, $1 \mathrm{BU}$ trophozoites appeared within $24 \mathrm{~h}$ including central and peripheral areas of the agar plate and after $72 \mathrm{~h}$, a considerable amount of trophozoites could be seen (Figure 4). After 5 weeks of cultivation, a number of cysts appeared in central and peripheral parts of the agar plate in controls. Notably, a similar kinetic was observed with MF-treated trophozoites, indicating that this drug neither substantially affected the ability of trophozoites to migrate on the agar plate, nor was encystment markedly affected (Figure 4). Although MF can diminish the trophozoite amount (Figure 2) and result in the highest amount of LDH release (Figure 1), the surviving cells are still viable.

However, when PHMB- and PD-treated 1BU trophozoites were spotted onto the non-nutrient agar E. coli plates, respectively, and monitored for growth and encystment, a more cohesive picture to those seen with the aforementioned assays emerged (Figure 4). Small, strange shaped trophozoites appeared on central and peripheral parts of the agar plate after $72 \mathrm{~h}$ of cultivation (Figure 5). Nevertheless, after 5 weeks of cultivation, no fresh cysts were observed at central or peripheral parts of the agar plate. It may indicate that the ability of encystment was hampered by the drug treatment.

When put into relation with the trypan blue assay results and the calcein-AM/ethidium homodimer-1 stainings (Figures 1 and 2), one can furthermore come to the conclusion that a positive staining of 1BU trophozoites for trypan blue or ethidium homodimer-1 does not allow to state that all these cells are dead. This conclusion is also strengthened by the findings that considerable amounts of PD- and PHMBtreated 1BU trophozoites were also positive for calcein, indicating that these cells were intact enough to allow the conversion of calcein-AM to calcein. In addition, PHMB- and PD-treated trophozoites released a lower LDH amount. Nevertheless, the high percentage of trypan bluepositive PHMB- and PD-treated trophozoites may refer to a damage of the cell membrane, which may also be the reason of their inability to form normal shaped trophozoites and encyst again.

When PHMB- and PD-treated trophozoites were monitored after 5 weeks of growth on non-nutrient agar E. coli plates, no cysts were found at the central or peripheral parts of the agar plates (Figure 4). This suggests that PHMB and PD interfere with the capacity of $A$. castellanii trophozoites to form cysts under unfavorable conditions (Figure 4).

When drug-treated cysts were tested with the nonnutrient agar E. coli assay, the following observations were made (Figure 5): in the control and the MF-treated 1BU cyst groups, trophozoites became visible on central and peripheral parts of the agar plate after $24 \mathrm{~h}$ of cultivation (Figure 5). These findings indicate that $A$. castellanii $1 \mathrm{BU}$ cysts can germinate on non-nutrient agar $E$. coli plates and migrate on the agar, and that these abilities were not markedly affected 
Figure 6. In 0.02\% PHMB- and 0.1\% PD-treated 1BU cyst groups, strange shaped trophozoites appeared after $72 \mathrm{~h}$, which did not form cysts again following 5 weeks. The strange trophozoites were smaller and thinner compared to the normal trophozoites

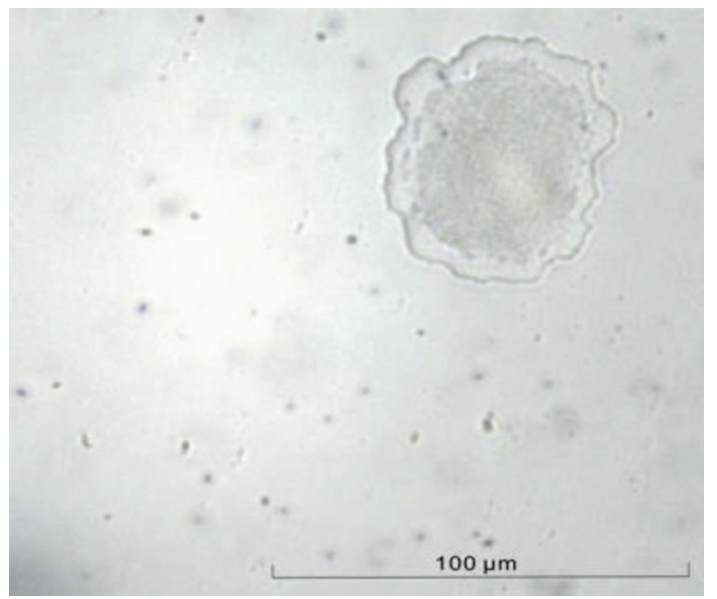

by the MF treatment. Unfortunately, we were unable to determine the percentage rates of $1 \mathrm{BU}$ excystment, as empty cysts were not clearly distinguishable from filled cysts by our light microscopy approach. After 5 weeks of cultivation, cysts could be observed in the central and peripheral parts of the agar plates, showing that MF-treated cysts did not lose their encystment abilities (Figure 5).

A different picture emerged, when PHMB- and PD-treated cysts were tested with this assay (Figure 5). In both drug-treated groups, irregularly shaped, smaller trophozoites with a thinner cell wall appeared after $72 \mathrm{~h}$ in the central and peripheral parts of the agar plates, which did not form cysts again within the 5 weeks of monitoring.

After 5 weeks of incubation, the appearance of roundish but flat cells with a discontinuous double wall was noticed in the PD- and PHMB-treated cyst groups (Figure 6). The amount of these irregularly shaped trophozoites or cysts increased over time and was also observed in the central and peripheral parts of the agar plates. These findings indicate that both drugs substantially interfered with the viability of $A$. castellanii $1 B U$ cysts and the ability to form regular trophozoites, supporting the idea that $\mathrm{PHMB}$ and $\mathrm{PD}$ are promising drug candidates for the treatment of $\mathrm{AK}$, while MF seems to have only a reduced effect on the viability of $A$. castellanii trophozoites and cysts.

\section{DISCUSSION AND CONCLUSIONS}

Acanthamoeba species can develop in two life forms, trophozoites and cysts. To be effective against these protozoan pathogens, a drug should also attack the dormant state of the pathogen, and as a consequence, efficacy of an antiamoebic drug should be evaluated with both life forms. In this study, we tested a number of common enzymatic- and dye-based viability assays to study the killing efficacies of antiamoebic drugs against $A$. castellanii $1 \mathrm{BU}$ trophozoites and cysts.
When compared with the non-nutrient agar E. coli plate assay, which served as gold standard in this comparison, none of the enzymatic- and dye-based assays tested in this study yielded results with both life forms that were comparable with the results obtained with the culture-based method. Discrepancies were especially evident with the drug MF, which yielded almost no effect on the viability of 1BU trophozoites and cysts when assayed with the culture-based method, while all enzymatic- and dye-based assays tested in this study suggested a good to excellent killing efficacy of this drug to at least one of the life forms of this pathogen. These findings suggest that commonly used viability assays such as trypan blue staining and life-dead staining with calcein-AM and ethidium homodimer-1 may - depending on the drug - overestimate the killing efficacy of the antiamoebic agent, while the culture-based non-nutrient agar E. coli plate assay proofed to be a reliable method to study the drug efficacy of antiamoebic agents against both life forms of this protozoan pathogen.

Conflict of Interest: The authors declare no conflict of interest. They assure that there are no links with a company whose product is mentioned in the article or a company that distributes a competing product. The authors also state that the presentation of the topic is independent and the presentation of the content is product-neutral.

\section{REFERENCES}

1. Siddiqui, R., Khan, N. A.: Biology and pathogenesis of Acanthamoeba. Parasit Vectors 5, 6 (2012).

2. Mazur, T., Hadaś, E., Iwanicka, I.: The duration of the cyst stage and the viability and virulence of Acanthamoeba isolates. Trop Med Parasitol 46, 106-108 (1995).

3. Aksozek, A., McClellan, K., Howard, K., Niederkorn, J. Y., Alizadeh, H.: Resistance of Acanthamoeba castellaniii cysts to physical, chemical, and radiological conditions. J Parasitol 88, 621-623 (2002).

4. Lorenzo-Morales, J., Khan, N. A., Walochnik, J.: An update on Acanthamoeba keratitis: Diagnosis, pathogenesis and treatment. Parasite 22, 10 (2015).

5. Geith, S., Walochnik, J., Prantl, F., Sack, S., Eyer, F.: Lethal outcome of granulomatous acanthamoebic encephalitis in a man who was human immunodeficiency virus-positive: A case report. J Med Case Rep 12, 201 (2018).

6. Doan, N., Rozansky, G., Nguyen, H. S., Gelsomino, M., Shabani, S., Mueller, W., Johnson, V.: Granulomatous amoebic encephalitis following hematopoietic stem cell transplantation. Surg Neurol Int 6, S459-S462 (2015).

7. Walochnik, J., Scheikl, U., Haller-Schober, E. M.: Twenty years of Acanthamoeba diagnostics in Austria. J Eukaryot Microbiol 62, 3-11 (2015).

8. Szentmáry, N., Daas, L., Matoula, P., Goebels, S., Seitz, B.: Akanthamöbenkeratitis [Acanthamoeba keratitis]. Ophthalmologe 110, 1203-1210 (2013).

9. Szentmáry, N., Daas, L., Shi, L., Laurik, K. L., Lepper, S., Milioti, G., Seitz, B.: Acanthamoeba keratitis - Clinical signs, 
differential diagnosis and treatment. J Curr Ophthalmol 31, 16-23 (2018).

10. Shi, L., Stachon, T., Seitz, B., Wagenpfeil, S., Langenbucher, A., Szentmáry, N.: The effect of antiamoebic agents on viability, proliferation and migration of human epithelial cells, keratocytes and endothelial cells, in vitro. Curr Eye Res 43, 725-733 (2018).

11. Lee, X., Reimmann, C., Greub, G., Sufrin, J., Croxatto, A.: The Pseudomonas aeruginosa toxin L-2-amino-4-methoxy-trans-3butenoic acid inhibits growth and induces encystment in Acanthamoeba castellaniii. Microbes Infect 14, 268-272 (2012).

12. Siddiqui, R., Abjani, F., Yeo, C. I., Tiekink, E. R., Khan, N. A.: The effects of phosphanegold (I) thiolates on the biological properties of Acanthamoeba castellaniii belonging to the T4 genotype. J Negat Results Biomed 16, 6 (2017).

13. Mito, T., Suzuki, T., Kobayashi, T., Zheng, X., Hayashi, Y., Shiraishi, A., Ohashi, Y.: Effect of photodynamic therapy with methylene blue on Acanthamoeba in vitro. Invest Ophthalmol Vis Sci 53, 6305-6313 (2012).

14. Chen, Z., Xuguang, S., Zhiqun, W., Ran, L.: In vitro amoebacidal activity of photodynamic therapy on Acanthamoeba. Br J Ophthalmol 92, 1283-1286 (2008).

15. Ash, L. R., Orihel, T. C.: Proteose Peptone-Yeast ExtractGlucose Medium. Parasites, a Guilde to Laboratory Procedures and Identification. American Sociesty of Clinical Pathologist, Chicago, IL, 1987, pp. 134-135.

16. Neff, R. J., Ray, S. A., Benton, W. F., Wilborn, M.: Induction of synchronous encystment (differentiation) in Acanthamoeba sp. In Prescott D. M. (ed): Methods in Cell Physiology, Volume 1. Academic Press Inc., New York, 1964, pp. 55-83.

17. Page, F. C.: A New Key to Freshwater and Soil Gymnamoebae. Freshwater Biological Association, Ambleside, Cumbria, UK, 1988, p. 122.
18. Narasimhan, S., Madhavan, H. N., Therese, T.: Development and application of an in vitro susceptibility test for Acanthamoeba species isolated from keratitis to polyhexamethylene biguanide and chlorhexidine. Cornea 21, 203-205 (2002).

19. Kowalski, R. P., Abdel Aziz, S., Romanowski, E. G., Shanks, R. Q., Nau, A. C., Raju, L. V.: Development of a practical complete-kill assay to evaluate anti-acanthamoeba drugs. JAMA Ophthalmol 131, 1459-1462 (2013).

20. Monk, I. R., Tree, J. J., Howden, B. P., Stinear, T. P., Foster, T. J.: Complete bypass of restriction systems for major Staphylococcus aureus lineages. MBio 6, e00308-15 (2015).

21. Sunada, A., Kimura, K., Nishi, I., Toyokawa, M., Ueda, A., Sakata, T., Suzuki, T., Inoue, Y., Ohashi, Y., Asari, S., Iwatani, Y.: In vitro evaluations of topical agents to treat Acanthamoeba keratitis. Ophthalmology 121, 2059-2065 (2014).

22. Siddiqui, R., Aqeel, Y., Khan, N. A.: The development of drugs against Acanthamoeba infections. Antimicrob Agents Chemother 60, 6441-6450 (2016).

23. Strober, W.: Trypan blue exclusion test of cell viability. Curr Protoc Immunol 21, A.3B.1-A.3B32 (2001).

24. Crowley, L. C., Marfell, B. J., Waterhouse, N. J.: Analyzing cell death by nuclear staining with Hoechst 33342. Cold Spring Harb Protoc 2016 (2016).

25. Eddaoudi, A., Canning, S. L., Kato, I.: Flow cytometric detection of G0 in live cells by Hoechst 33342 and Pyronin Y staining. Methods Mol Biol 1686, 49-57 (2018).

26. Romano, V., Parekh, M., Ruzza, A., Willoughby, C. E., Ferrari, S., Ponzin, D., Kaye, S. B., Levis, H. J.: Comparison of preservation and transportation protocols for preloaded Descemet membrane endothelial keratoplasty. Br J Ophthalmol 102, 549-555 (2018).

27. Chan, L. L., McCulley, K. J., Kessel, S. L.: Assessment of cell viability with single-, dual-, and multi-staining methods using image cytometry. Methods Mol Biol 1601, 27-41 (2017). 